\title{
On Multi-Utility Representation of Equitable Intergenerational Preferences
}

\author{
Kuntal Banerjee and Ram Sewak Dubey
}

\begin{abstract}
We investigate the possibility of representing ethical intergenerational preferences using more than one utility function. It is shown that the impossibility of representing intergenerational preferences equitably persists in the multi-utility frame work with some resonable restrictions on the cardinality of the set of utilities.
\end{abstract}

\section{Introduction}

In ranking infinite utility streams we seek to satisfy two basic principles. The equal treatment of all generations and the sensitivity of the ranking to the utility of every generation in the Pareto sense. The former is captured in the axiom of anonymity while the latter axiom is called strong Pareto. We will call a social evaluation satisfying these two conditions ethical. The theory of intergenerational social choice explores the possibility of obtaining ethical social evaluation criteria. We will not attempt to summarize the vast literature on intergenerational social choice, interested readers are referred to Basu and Mitra (2007) and the references therein.

Diamond (1965) established the impossibility of ranking infinite utility streams satisfying anonymity, strong Pareto and continuity of the Social Welfare Relation (SWR, a reflexive and transitive binary relation). Svensson (1980) showed that Diamond's impossibility result could be avoided by weakening the continuity requirement on the ethical Social Welfare Relation.

\footnotetext{
Kuntal Banerjee

Barry Kaye Collage of Business, Department of Economics, Florida Atlantic University, Boca Raton, USA. e-mail: kbanerje@fau.edu

Ram Sewak Dubey

Department of Economics, Cornell University, Ithaca, New York, USA.

e-mail: rsd28@cornell.edu
} 
While much of this literature concerned itself with the existence of ethical Social Welfare Orders (SWOs), Basu and Mitra (2003) proved that there is no ethical social welfare function.

In view of these impossibility results subsequent analysis was concentrated on defining ethical SWRs and exploring some of their important properties ${ }^{1}$.

Our concern in this paper is to investigate whether we can avoid the impossibility result of Basu and Mitra (2003) using some weaker requirement of representability. Two directions are pursued. For an ethical SWR we ask whether there is a RichterPeleg Representation of the partial order. It follows in a straightforward way from the analysis in Basu and Mitra (2003) that no such ethical SWR exists.

Following some recent developments in the theory of representable partial orders we ask whether one can define an ethical SWR that can be represented by not just a single utility function but possibly many utility functions. This approach is called the multi-utility representation ${ }^{2}$. As is argued by Ok (2002), in the special case with a multi-utility representation using a finite set of utility functions one might even be able to use the theory of vector optimization (multi-objective programming) in determining best alternatives over a constrained set, as is often the primary goal of most economic actors endowed with preferences. This feature makes this approach particularly appealing. The literature on multi-utility representation of binary relations have received significant attention in the works of Ok (2002), Ok and Evren (2007). Unfortunately, both the alternative approaches fail to yield a positive resolution to the Basu-Mitra impossibility result.

Preliminaries, are provided in the Section 2. In Section 3 the main results are stated and proofs are provided.

\section{Preliminaries}

The space of utility profiles (we will also call them utility streams) is the infinite cartesian product of the $[0,1]$ interval, denoted by $X^{3}$. Denoting by $\mathbb{N}$ the set of all natural numbers, we can write $X$ as $[0,1]^{\mathbb{N}}$. A partial order on any set is a binary relation $\succsim$ that is reflexive and transitive. The word partial order is used interchangeably with social welfare relation. The asymmetric ("strictly better than") and the symmetric ("indifferent to") relation associated with $\succsim$ will be denoted by $\succ$ and $\sim$ respectively. We will be concerned with the representation of social welfare relations that satisfy the following axioms. A SWR $\succsim$ defined on $X$ satisfies

Anonymity: For all $x, y \in X$, if there exists $i, j \in \mathbb{N}$ such that $x_{i}=y_{j}, x_{j}=y_{i}$ and $x_{k}=y_{k}$ for all $k \neq i, j$, then $x \sim y$.

\footnotetext{
${ }^{1}$ Asheim and Tungodden (2004), Banerjee (2006), Basu and Mitra (2007) and Bossert, Sprumont and Suzumura (2007) are some of the representative papers in this area.

${ }^{2}$ A precise definition of each approach is provided in Section 2.

${ }^{3}$ We will write a vector $x$ in $X$ or $\mathbb{R}^{\infty}$ as $\left(x_{1}, x_{2}, \ldots, x_{i}, \ldots\right)$. The following vector inequalities are maintained throughout this paper. $x>y$ iff $x_{i} \geq y_{i}$ for all $i$ and $x_{j}>y_{j}$ for some $j, x \geq y$ iff $x_{i} \geq y_{i}$ for all $i$. So, $x>y$ iff $x \geq y$ and $x \neq y$.
} 
Strong Pareto: For $x, y \in X$, if $x>y$, then $x \succ y$.

Social Welfare Relations that satisfy the axioms of anonymity and strong Pareto will be called ethical. To ease the writing, for any two sets $A, B$, let us denote by $A_{B}$ the class of functions with domain $A$ and range in $B$. Let us recall the standard notion of representing binary relations that are complete. Given $\succsim$, a SWO on a set $X$, we say that $u \in X_{\mathbb{R}}$ represents if $x \succsim y$ iff $u(x) \geq u(y)$. In this case, the order is said to have a standard representation.

A SWR on $X$ is said to have a Richter-Peleg Representation if there exists some $u \in X_{\mathbb{R}}$ such that $x \succ y$ implies $u(x)>u(y)$. It is easily seen that if $u$ is a RichterPeleg Representation of a partial order, then if $u(x)>u(y)$ holds for the pair $x, y$ we know that $y \succ x$ is not true, but we cannot conclude whether $x \succ y$ is true or false. So there is no way to recover the binary relation $\succ$ using the information in the Richter-Peleg Representation. This point is made in Majumdar and Sen (1976).

A SWR $\succsim$ on $X$ is said to have a multi-utility representation if there is some class $U \subset X_{\mathbb{R}}$ such that

$$
x \succsim y \text { iff } u(x) \geq u(y) \text { for all } u \in U .
$$

Obviously if a SWO has a standard representation, then it must have a multiutility representation, but the converse is not true. The multi-utility representation approach in utility theory has received significant attention through the works of Ok (2002), Ok and Evren (2007) and Mandler (2006). We use the term multi-utility representation to refer to this representation approach following Ok (2002). Notably, Mandler (2006) calls the class $U$, a psychology.

\section{Results}

It is now well known from the result in Basu and Mitra (2003) that ethical SWOs cannot have a standard representation. In this section, we will consider the representation of ethical SWRs on $X$ under the Richter-Peleg criterion and the multi-utility criterion. For ready reference let us state the Basu and Mitra (2003, Theorem 1).

Theorem 1 (Basu-Mitra Impossibility Theorem). There does not exist an ethical SWO in $X$ that has a standard representation.

As an easy consequence of this theorem it follows that an ethical SWR cannot have a Richter-Peleg Representation (RPR).

Proposition 1. There does not exist an ethical SWR that has a Richter-Peleg Representation.

Proof: Let $\succsim$ be an ethical SWR with its asymmetric and symmetric parts $\succ$ and $\sim$ respectively. Using the ethical SWR $\succsim$ and its RPR $u \in X_{\mathbb{R}}$ we can construct the following SWO: For $x, y \in X$, we define $\succsim^{\prime}$ by declaring $x \succsim^{\prime} y$ iff $u(x) \geq u(y)$. We will show that $\succsim^{\prime}$ is an ethical SWO. For any $x, y \in X$ satisfying $x>y$ we would have from strong Pareto, $x \succ y$. By the RPR of the SWR, we must have $u(x)>u(y)$, 
this implies from the definition of $\succsim^{\prime}, x \succ^{\prime} y$. Similarly, for any $x, y \in X$ with $x_{i}=y_{j}$, $x_{j}=y_{i}$ and $x_{k}=y_{k}$ for all $k \neq i, j$, we must have $x \sim y$. This means both $x \succ y$ and $y \succ x$ must be false, implying from the definition of RPR that $u(x)=u(y)$, implying $x \sim^{\prime} y$. This establishes that $\succsim^{\prime}$ is an ethical SWO and that $u$ is a standard representation of $\succsim^{\prime}$, thereby contradicting Theorem 1 .

We now turn our attention to multi-utility representation of ethical preferences. Suppose $\succsim$ is a SWR satisfying anonymity and strong Pareto. Assume that $\succsim$ has a multi-utility representation using a class of utility functions $U$. Let $x>y$, then $x \succ y$ as $\succsim$ satisfies strong Pareto. This implies that

$$
\text { for all } u \in U, u(x) \geq u(y) \text { and for some } u \in U, u(x)>u(y) \text {. }
$$

If for $x, y \in X$, there exists $i, j \in \mathbb{N}$ such that $x_{i}=y_{j}, x_{j}=y_{i}$ and $x_{k}=y_{k}$ for all $k \neq i, j$, then $x \sim y$. This implies

$$
u(x)=u(y) \text { for all } u \in U .
$$

Observe that in Proposition 1 in Ok and Evren (2007) it is shown that any partial order has a multi-utility representation (without restricting the cardinality of the set of utility function $U$ ). However, for the resultant representation to be tractable and useful we would prefer the utility set $U$ to be of minimal cardinality.

In that regard, given an ethical SWR $\succsim$ on $X$, we ask whether there is a multiutility representation with the set of utilities $U$ having finite cardinality? The answer to that is, no!

Suppose there is a multi-utility representation of $\succsim$ with the set of utility $U$ having cardinality 2 . Write $U=\left(u_{1}, u_{2}\right)$. Consider the function $u \in X_{\mathbb{R}}$ defined by $u(x)=$ $u_{1}(x)+u_{2}(x)$ and define a SWO $\succsim^{*}$ by $x \succsim^{*} y$ iff $u(x) \geq u(y)$. It is easily checked that $\succsim^{*}$ satisfies the axioms of anonymity and Strong Pareto. The function $u$ is also a standard representation of $\succsim^{*}$. This contradicts the conclusion of Theorem 1 . This contradiction establishes that no ethical SWR can have a multi-utility representation, where the cardinality of the set of utility functions is 2 . The idea of the proof readily extends to the case when the set $U$ is allowed arbitrary finite cardinality.

We can in fact show a stronger result. In the next theorem, it is shown that there is no ethical SWR that has a multi-utility representation using a set of utility functions that is countably infinite.

Theorem 2. There does not exist an ethical SWR that has a multi-utility representation with the set of utilities being countably infinite.

Proof: By way of contradiction, assume that there exists a SWR $\succsim$ that has a multiutility representation with the cardinality of the set of utilities $U$ being countably infinite. This is equivalent to saying that there exists a $u \in X_{\mathbb{R}^{\infty}}$ such that $x \succsim y$ iff $u(x) \geq u(y)$. Let $I$ denote the interval $[-1,1]$. Let $g: \mathbb{R}^{\infty} \rightarrow I^{\infty}$ be defined as follows:

$$
g_{i}(a)=\left\{\begin{array}{l}
\frac{a_{i}}{1+a_{i}} \text { if } a_{i} \geqslant 0 \\
\frac{a_{i}}{1-a_{i}} \text { if } a_{i}<0
\end{array} \text { for all } i \in \mathbb{N} \text { and all } a \in \mathbb{R}^{\infty}\right.
$$


and $g(a)=\left(g_{1}(a), g_{2}(a), \ldots.\right) \in I^{\infty}$.

Observe the following facts about the function $g$ : (a) $g_{i}(a)=0$ iff $a_{i}=0$ (b) $a_{i} /\left(1+a_{i}\right)$ is a strictly increasing function for all $a_{i} \geq 0$ and (c) $a_{i} /\left(1-a_{i}\right)$ is a strictly increasing function for all $a_{i}<0$. Define the vector $\alpha=\left(1 / 2,1 / 2^{2}, \ldots\right)$ and a function $V: X \rightarrow \mathbb{R}$ as follows :

$$
V(x)=\alpha \cdot g(u(x)) \cdot^{4}
$$

Let us now define the SWO as follows: for all $x, y \in X$

$$
x \succsim^{\prime} y \text { iff } V(x) \geq V(y) .
$$

We will now show that $\succsim^{\prime}$ satisfies the axioms of anonymity and strong Pareto.

To check anonymity of $\succsim^{\prime}$, let $x \in X$ and $x_{\pi}$ be a profile with the utilities of the $i^{t h}$ and $j^{t h}$ generation in $x$ swapped. By (3), $u(x)=u\left(x_{\pi}\right)$ and consequently, $g(u(x))=g\left(u\left(x_{\pi}\right)\right)$. Hence, $V(x)=V\left(x_{\pi}\right)$. So, $x \sim^{\prime} y$.

To check strong Pareto, let $x, y \in X$ such that $x>y$. We will show that $V(x)>$ $V(y)$. By (2), $u_{i}(x) \geq u_{i}(y)$ for all $i \in \mathbb{N}$ and for at least some $j \in \mathbb{N}, u_{j}(x)>u_{j}(y)$. Three cases are possible: (i) $u_{i}(x) \geq u_{i}(y) \geq 0$ (ii) $u_{i}(x) \geq 0 \geq u_{i}(y)$ and (iii) $0 \geq$ $u_{i}(x) \geq u_{i}(y)$. In case (i), $g_{i}\left(u_{i}(x)\right) \geq g_{i}\left(u_{i}(y)\right) \geq 0$ follows from (4) and the fact that $g_{i}(u)$ is a strictly increasing function in $u_{i} \geq 0$. In case (ii), $g_{i}\left(u_{i}(x)\right) \geq 0 \geq$ $g_{i}\left(u_{i}(y)\right)$ follows from the definition of $g$. In case (iii), $0 \geq g_{i}\left(u_{i}(x)\right) \geq g_{i}\left(u_{i}(y)\right)$ follows from (4) and the fact that $g_{i}(u)$ is a strictly increasing function in $u_{i}<$ 0 . Observe that since each component function in the definition of $g_{i}$ is strictly increasing, $g_{j}\left(u_{j}(x)\right)>g_{j}\left(u_{j}(y)\right)$.

In all three cases, $g_{i}\left(u_{i}(x)\right) \geq g_{i}\left(u_{i}(y)\right)$, and $g_{j}\left(u_{j}(x)\right)>g_{j}\left(u_{j}(y)\right)$. From the definition of $V$ it now follows that $V(x)>V(y)$. This implies $x \succ^{\prime} y$.

So $\succsim^{\prime}$ is a SWO that has a standard representation satisfying anonymity and strong Pareto. This violates Theorem 1.

Acknowledgements We thank Tapan Mitra for a helpful conversation.

\section{References}

1. Asheim G.B, Tungodden B. Resolving distributional conflicts between generations, Econ. Theory 24 (2004), 221-230

2. Banerjee K. On the extension of utilitarian and Suppes-Sen social welfare relations to infinite utility streams, Soc. Choice Welfare 27 (2006), 327-339

3. Basu K, Mitra T. Aggregating infinite utility streams with intergenerational equity: the impossibility of being Paretian, Econometrica 71 (2003), 1557-1563

4. Basu K, Mitra T. Utilitarianism for infinite utility streams: a new welfare criterion and its axiomatic characterization, J. Econ. Theory 133 (2007), 350-373

5. Bossert W, Sprumont Y, Suzumura K. Ordering infinite utility streams. J. Econ. Theory 135 (2007), 579-589

${ }^{4} \alpha \cdot g(u(x))=\sum_{i \in \mathbb{N}}(1 / 2)^{i} g_{i}(u(x))$. 
6. Diamond P.A. The evaluation of infinite utility streams. Econometrica 33 (1965), 170-177

7. Majumdar M, Sen A. A note on representation of partial orderings, Rev. Econ. Studies 43 (1976), 543-545

8. Mandler M. Cardinality versus ordinality: a suggested compromise, Amer. Econ. Review 96 (2006), 1114-1136

9. Ok E. Utility Representation of an incomplete preference relation, J. Econ. Theory 104 (2002), 429-449

10. Ok E, Evren O. On the multi-utility representation of preference relations, mimeo New York University (2007)

11. Svensson L.G. Equity among generations. Econometrica 48 (1980), 1251-1256 